\title{
Citrobacter koseri meningitis in a special care baby unit
}

\author{
C. D. RIBEIRO, P. DAVIS, AND D. M. JONES \\ From the Department of Bacteriology, Withington Hospital, Manchester M20 8LR
}

SYNOPSIS An outbreak of meningitis due to Citrobacter koseri in a special care baby unit is described. The organism showed a high capacity for spread among the babies on the unit and although the intestinal carriage rate was high, the clinical case:carrier ratio was low.

The occurrence of a small number of cases of meningitis due to Citrobacter koseri (syn C. diversus, Levinea malonatica) has been reported (Gross et al, 1973; Gwynn and George, 1973; Duhamel et al, 1975; Tamborlane and Soto, 1975). All the cases have occurred in young babies, mostly in the first two weeks of life, and the babies have nearly all been premature. Of the 11 cases described, eight have occurred in two outbreaks in premature or special care baby units (Gross et al, 1973; Gwynn and George, 1973). In both these outbreaks the cases occurred over long periods of time, six and nine months respectively. The babies were severely ill and the prognosis of such infections seems to be poor.

We have observed a similar outbreak involving three cases and have made some observations on the epidemiology of the infections.

\section{Bacteriology}

The strains of $C$. koseri were catalase positive, oxidase negative, motile, Gram-negative rods. They fermented glucose with the production of acid and gas, sucrose, maltose, mannitol, dulcitol, adonitol, sorbitol, trehalose, cellobiose, galactose, and arabinose, but not lactose, salicin, inositol, rhamnose, xylose or raffinose. They were methyl red positive and Voges-Proskauer negative and produced indole from tryptone water. They did not grow in the presence of potassium cyanide. $\mathrm{H}_{2} \mathrm{~S}$ was not produced in Kligler's iron agar, Simmons' citrate was utilized, urease was detected in Christensen's urea at 48 hours, and gelatin was not liquefied in 10 days. We considered that the biochemical characters were consistent with the definition of $C$. koseri.

The minimal inhibiting concentrations of various

Received for publication 3 May 1976 antibiotics for the citrobacter were: chloramphenicol $>100 \mathrm{mg} / \mathrm{l}$; gentamicin $0.6 \mathrm{mg} / \mathrm{l}$; cephalexin $20 \mathrm{mg} / \mathrm{l}$; ampicillin $>20 \mathrm{mg} / \mathrm{l}$; carbenicillin $>400 \mathrm{mg} / \mathrm{l}$. As the citrobacter was carbenicillin-resistant, we used a selective medium containing carbenicillin $(100 \mathrm{mg} / \mathrm{l})$ and adonitol $(1 \%)$ to isolate the organism, particularly from faecal specimens. In some of the excreters the citrobacter was enumerated in the faeces.

The outbreak

Three cases of meningitis due to $C$. koseri occurred between March and August 1973 in a special care baby unit.

\section{CASE 1}

A female infant who was born by assisted breech delivery at 36 weeks' gestation. Birth weight was $6 \mathrm{lb}$ $1 \frac{1}{2} \mathrm{oz}$. She became pyrexial on the 10th day of life ( 2 March 1973). Treatment was begun with intramuscular ampicillin and cloxacillin. The next day the baby was febrile and had convulsions. The cerebrospinal fluid was cloudy with raised protein and low sugar, and the white cell count $1427 / \mathrm{mm}^{3}(90 \%$ polymorphs); Gram-negative rods were seen in the film. Intensive treatment with ampicillin and gentamicin, together with general resuscitative measures including ventilation, failed to save the baby who died on the 12th day. $C$. koseri was grown from both CSF and blood cultures ante mortem.

\section{CASE 2}

This case occurred 15 days after the first. This baby was female and born at $31 / 32$ weeks' gestation weighing $3 \mathrm{lb} 10 \mathrm{oz}$. Delivery was normal. She suffered from a mild respiratory distress syndrome but was reasonably well to the eighth day of life (17 March 1973) when she was seen to be floppy, 
cyanosed, unresponsive, and suffering from apnoeic attacks. The CSF was cloudy with $985 \mathrm{WBC} / \mathrm{mm}^{3}$ ( $98 \%$ polymorphs); Gram-negative rods were seen in the film. The baby was severely acidotic and, despite intensive treatment including intravenous benzyl penicillin and intravenous gentamicin, she collapsed and died. C. koseri was isolated from CSF, blood culture, and nasal and rectal swabs ante mortem, and from blood, lung, liver, brain, and gut post mortem.

With the occurrence of a second C. koseri infection a comprehensive microbiological investigation of the special care baby unit was begun. Culture of the faeces and umbilical and throat swabs of the babies showed that nearly all (7/8) were colonized with the organism. The alimentary tract was the main site of the colonization in these babies but in over half $(4 / 7)$ the organism was grown from the umbilicus. None of the babies' mothers or members of the staff was found to be a faecal carrier of the organism. Careful cultural study of all parts of the environment of the unit, including sinks and humidifiers, failed to demonstrate the presence of the organism, except on one occasion when a single colony was grown on a settle plate placed in a room where an infected baby was being nursed. All the medicaments in use on the unit and a selection of the milk feeds prepared for the unit were cultured with negative results.

With such widespread colonization of the babies, admissions to the unit were stopped. Despite the most careful handling of the colonized babies and isolation of these within the unit, cross-infection by the citrobacter was not controlled. Eventually the majority of babies were discharged but a small number who required prolonged inpatient care were established in a separate ward. Those babies who were colonized were nursed by different staff members from those who were not, and all the babies were subsequently transferred either home or to isolation elsewhere on 4 June 1973. After cleaning and disinfection the special care unit was reopened to admissions on 14 June 1973.

It was clear by this time that the clinical attack rate was very low compared with the colonization rate. The rates of colonization relating to the month of admission to the unit up to this time are shown in the figure. The figures include babies examined at the follow-up clinic who had been present in the unit before the birth of the first clinical case. The isolation of the citrobacter from these babies was presumptive evidence that the organism had been present among the babies nursed on the unit before the occurrence of the first case.

After the unit was reopened we continued to examine routine faecal specimens and the citrobacter was again isolated from the babies but no carriers were found among the staff. Despite efforts to con-

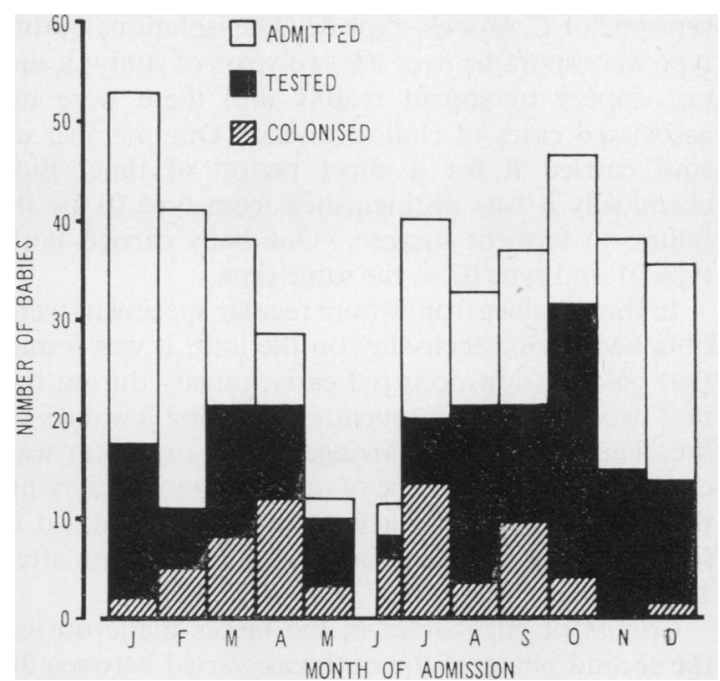

Figure Citrobacter koseri colonization of babies admitted to special care unit during 1973.

trol cross-infection some babies on the unit were colonized during the next six months (see figure), and in this time one further clinical case of meningitis occurred.

\section{CASE 3}

This baby was the second of female twins. Her birth weight was $4 \mathrm{lb} 7 \mathrm{oz}$ and her condition at birth was good. She became ill on the eighth day of life (4 August 1973) with fever and a swollen abdomen. A lumbar puncture confirmed the diagnosis of bacterial meningitis, and $C$. koseri was subsequently grown. Intensive antibiotic and supportive treatment was begun very soon after the onset of symptoms; the child survived but was left with a considerable neurological defect. This child has since died of an unrelated infection and at the post-mortem examination there was considerable cerebral necrosis.

We continued to screen for $C$. koseri throughout 1974 but did not isolate it from any of the babies admitted to the unit after December 1973. Altogether 65 babies from the special care baby unit were found to be colonized at some time during the investigation. Citrobacter strains isolated from the clinical cases and from a proportion of the colonized babies were serotyped by $\mathrm{Dr}$ B. Rowe at the Salmonella and Shigella Reference Laboratory. The strains associated with this outbreak of infection were all type 01 as described in the antigenic schemes for C. koseri (Gross and Rowe, 1974; 1975).

In the course of the investigation a small number of babies were found to be colonized by a different 
serotype of $C$. koseri-type 07 . The isolations of this type were sporadic over the two years of study; it did not appear to spread readily and there were no associated cases of clinical disease. One member of staff carried it for a short period of time. Biochemically it was distinguished from type 01 by its failure to ferment sucrose. One baby carried both type 01 and type 07 at the same time.

In those babies from whom regular specimens were obtained during their stay on the unit, it was found that colonization occurred early, mostly during the first two weeks and frequently in the first few days of life. The duration of carriage of the organism was extremely variable. Some of our babies were carrying the organism for only a few days; others carried it for several months, and one was still excreting after 14 months.

Counts of citrobacter in the faeces made during the second phase of the outbreak varied between 30 and $5 \times 10^{10}$ organisms per gram of faeces. Many of the normal excreters had counts of $10^{10}$ organisms per gram. Case 3 was found to have a count of $5 \times 10^{10}$ organisms per gram, but this was no different from her twin sibling whose faecal count was $1 \times 10^{10}$ organisms per gram and who did not become ill.

\section{Discussion}

Over a period of one year 65 babies were found to be infected with a particular serotype of $C$. koseri. The infected babies were widely colonized, the organisms being mainly isolated from the gut but also being found on the umbilicus. Three $(4.6 \%)$ of the colonized babies became ill with systemic invasion by the organism. Of these three cases, two died and one was left with residual neurological damage. This pattern of severe clinical illnesses is similar to that found by other authors (Gross et al, 1973; Gwynn and George, 1973; Duhamel et al, 1975; Tamborlane and Soto, 1975). By examining specimens from children seen in the outpatient clinic and who had been discharged from the unit before the birth of the first clinical case we were able to show that the organism had been present among the babies on the ward for some time previously. We were not able to determine the origin of the infection. This organism showed an ability to spread rapidly to a high proportion of the babies present-at one stage $75 \%$ of the babies admitted to the unit-in spite of an awareness of its presence and attempts to control it by isolation of babies within the unit. Spread was presumably by the faecal-oral route and, although the mode of transmission was not apparent, the possibility of some limited air spread would appear to be indicated by the positive settle plate. The cause of the re-emergence of the organism after closure of the ward and fumigation was not clear. Possibly the organism was being carried by a staff member although this was not detected. The spread of the citrobacter was eventually controlled by the continued policy of isolation. The duration of carriage of the organism in this outbreak was very variable, ranging from a few days to at least 14 months. There appeared to be no correlation between the faecal count of the organism and the likelihood of developing illness. It is of interest that although case 3 and her twin had almost identical faecal citrobacter counts, one became ill and the other did not. It could be inferred that natural maternal antibody is not important in preventing invasive infection since both babies presumably acquired the same maternal immunoglobulins.

The epidemiological pattern of spread of infection with this organism was similar to that exhibited by enteropathogenic $E$. coli in that very rapid transmission occurred among neonates once the organism had been introduced into a unit. Since at least four serotypes of $C$. koseri have been isolated from cases of meningitis-01, 02, 07, and 08 (Rowe et al, 1975) -pathogenicity does not seem to be related to a particular serotype. The behaviour of the 07 sero type observed during our investigations suggests that individual strains of the same serotype may differ in their ability to spread and in their capacity to cause disease.

We should like to thank Dr D. Macaulay for allowing us to study his patients and the staff of the special care baby unit and the baby clinic for providing us with the specimens.

\section{References}

Duhamel, M., Cuvelier, A., Cousin, J., and Fournier, A. (1975). Septicémie et méningite néo-natales a Levinea malonatica. Nouv. Presse méd., 4, 428.

Gross, R. J. and Rowe, B. (1974). The serology of Citrobacter koseri Levinea malonatica and Levinea amalonatica. J. med. Microbiol., 7, 155-161.

Gross, R. J. and Rowe, B. (1975). Citrobacter koseri I. An extended antigenic scheme for Citrobacter koseri (syn. $C$. diversus, Levinea malonatica). J. Hyg. (Lond.), 75, .121127.

Gross, R. J., Rowe, B., and Easton, J. A. (1973). Neonatal meningitis caused by Citrobacter koseri. J. clin. Path., 26, 138-139.

Gwynn, C. M. and George, R. H. (1973). Neonatal citrobacter meningitis. Arch. Dis. Childh., 48, 455-458.

Rowe, B., Gross, R. J., and Allen, H. A. (1975). Citrobacter koseri. II. Serological and biochemical examination of Citrobacter koseri strains from clinical specimens. J. Hyg. (Lond.), 75, 129-134.

Tamborlane, W. V., Jr. and Soto, E. V. (1975). Citrobacter diversus meningitis: a case report. Pediatrics, 55, 739-741. 